\title{
Effect of lysozyme subphase and insertion on several lipid films
}

\author{
Torrent-Burgués $\mathbf{J}^{1 *}$ and Raju $\mathbf{R}^{1,2}$ \\ ${ }^{1}$ Department of Chemical Engineering, Universitat Politècnica de Catalunya (UPC), C/ Colom 1, E08222 Terrassa, Barcelona, Spain \\ ${ }^{2}$ Centre for Molecular and Nanoscale Physics, School of Science, RMIT University Melbourne, Australia
}

\begin{abstract}
The influence of proteins on lipid monolayers is a subject of biological interest. In this work the influence of lysozyme on lipid films of stearic acid, oleic acid, cholesterol, DPPC and POPC has been studied. The Langmuir monolayer technique, using surface pressure-area isotherms and elastic modulus plots, as well as protein insertion experiments in lipid monolayers, have been used. Results indicate that lysozyme affects the lipid monolayer formation, the elastic modulus and, sometimes, the physical state of the monolayer. This influence is more important till moderate surface pressures. At high surface pressures and near the physiological value of lateral pressure of $33 \mathrm{mN} / \mathrm{m}$, there is expulsion of lysozyme out of the monolayer. This expulsion is more important for stearic acid, DPPC and POPC. The lower value of maximum insertion pressure is for stearic acid and the higher one is for oleic acid. The relation between the initial and the increased surface pressure in insertion experiments has been analysed.
\end{abstract}

\section{Introduction}

Fatty acids, cholesterol and phospholipids have been studied with the Langmuir technique because of their biological interest and they form ordered and compact monolayers. Interactions of proteins with lipid membranes and films are of great interest for biological systems as cellular membranes, tear films [1], and mimicking them for technological applications. Studies on surface properties of protein solutions are described elsewhere [2-11]. Similarly, some studies of the influence of proteins on lipid monolayers are those of references [1235]. The interpretation of protein influence and interactions with lipid monolayers has been studied in [5,36-40].

Only some of these cited works have studied lysozyme $[9,11]$ or the influence of lysozyme on lipid films $[17,18,26,27]$. On the other hand, the influence of lysozyme on liposomes are those of references [41-43]. Biological functions of lysozyme have been suggested to be determined by the lipid binding properties of this protein [41]. Several contributions have been proposed for that, including electrostatic and hydrophobic ones. Lysozyme is a tear film protein, with bacteriostatic functions among others, and its influence on Meibomian lipid films has been studied by Miano et al. [1] and Mudgil et al. [34], but Meibomian lipid films from tears are a complex mixture of lipids.

In this work the binding properties and insertion of lysozyme on lipid layers will be studied using the monolayer technique and analysed using the method proposed in reference [37]. The lipids selected are neutral, slightly ionised or zwitterionic, in order to discard neat charge contributions. The specific systems here proposed, that is the influence of lysozyme on lipid films of stearic acid, oleic acid, cholesterol, DPPC and POPC, which are different in nature (fatty acids, sterol, phospholipids), have not been studied previously. Some of them present an unsaturation on the fatty acid or in the phospholipid hydrocarbon chains, which permits to see the influence of unsaturations on the effect of lysozyme. The Langmuir monolayer technique using $P-A$ isotherms and compressibility plots, as well as protein insertion experiments in lipid monolayers, have been used since this technique permits the control of the surface pressure.

\section{Materials and methods}

\section{Materials}

Oleic acid (OA) was provided by Fluka and palmitoyloleoylphosphatidylcholine (POPC) was purchased from Avanti Polar Lipids. Stearic acid (SA), dipalmitoylphosphatidylcholine (DPPC), cholesterol (CHOL) and lysozyme (LYS) from chicken egg white were provided by Sigma-Aldrich. Chloroform of analytical grade from Sigma-Aldrich were used in lipid solution preparation. Water was ultrapure MilliQ ${ }^{\circ}(18.2 \mathrm{M} \Omega \cdot \mathrm{cm})$.

\section{Techniques}

Monolayer formation: Langmuir monolayer formation was carried on a trough (Nima Technology, Cambridge, UK) model 1232D1D2 equipped with two movable barriers. The surface pressure was measured using paper Whatman1 held by a Wilhelmy balance connected to a microelectronic system registering the surface pressure (П). The paper sheet was always cleaned and conditioned in the subphase prior measurements. In one case, the subphase used in these experiments was MilliQ quality water. In other case, the subphase used was a solution containing LYS (at a concentration of $0.1 \mathrm{~g} \cdot \mathrm{L}^{-1}$ ). Prior to the subphase addition, the trough was cleaned twice with chloroform and once with MilliQ quality water. Residual impurities were cleaned

*Correspondence to: J Torrent-Burgués, Department of Chemical Engineering, Universitat Politècnica de Catalunya (UPC), C/ Colom 1, E08222 Terrassa, Barcelona, Spain, E-mail: juan.torrent@upc.edu

Key words: elastic modulus, lipid monolayers, lysozyme insertion, maximum insertion pressure, surface pressure-area isotherm

Received: April 03, 2019; Accepted: April 22, 2019; Published: April 25, 2019 
from the air-liquid interface by surface suctioning. Solutions of the lipids were prepared using chloroform (at concentrations of 0.5 $\left.\mathrm{mg} \cdot \mathrm{mL}^{-1}\right)$ and an aliquot of $100 \mu \mathrm{L}$ was spread at the air-liquid interface using a high precision Hamilton microsyringe. Barrier closing rates were fixed at $50 \mathrm{~cm}^{2} \cdot \mathrm{min}^{-1}$. Surface pressure-area isotherm were recorded, in one case, for the LYS subphase without lipid addition, and in the other case recording was carried out adding the lipid solution drop by drop to the subphase, waiting 15 minutes for perfect spreading and solvent evaporation. $\mathrm{X}$-axis of the isotherm plot represents the area per molecule. Experiments were conducted at $22 \pm 1^{\circ} \mathrm{C}$.

\section{Insertion experiments}

The insertion of LYS into lipid monolayers was measured using a NIMA Langmuir Film Balance equipped with a Wilhelmy plate (Nima Technology, Coventry) and a small Teflon trough that was rinsed with chloroform and distilled water before use. All experiments were performed at $22 \pm 1^{\circ} \mathrm{C}$.

For these experiments, a lipid stock solution was prepared and added drop wise on the water subphase until the desired lipid pressure was achieved. After $15 \mathrm{~min}$, when the equilibrium of the lipid monolayer was reached, a LYS solution was injected into the water subphase to attain a concentration of $0.1 \mathrm{~g} \cdot \mathrm{L}^{-1}$. The surface pressure changes were monitored as a function of time during $15 \mathrm{~min}$. For a blank experiment the same procedure was followed but without using lipid.

\section{Results and discussion}

\section{Surface lysozyme behaviour}

In this section the surface behaviour of LYS is reported.

At first the surface tension of the subphase with $0.1 \mathrm{~g} \cdot \mathrm{L}^{-1}\left(6.9410^{-6}\right.$ M) LYS solution was measured, obtaining a value of $71.0 \mathrm{mN} / \mathrm{m}$. This value is only slightly lower than that of water $\left(72.6 \mathrm{mN} / \mathrm{m}\right.$ at $\left.22^{\circ} \mathrm{C}\right)$. Also, a value of $\mathrm{pH}=5.0$ was measured for this solution, which is only slightly more acidic than water $(\mathrm{pH}=6.0)$. A fresh LYS solution of $0.1 \mathrm{~g} \cdot \mathrm{L}^{-1}$ have shown a value of zero for the surface pressure when recording an isotherm (Figure 1). A similar behaviour was observed for asparaginase [44] on its aqueous subphase, during compression.

\section{Influence of lysozyme over lipid monolayer isotherms.}

The presence of LYS in the subphase and its influence on lipid monolayers has been investigated. The lipids used are DPPC, POPC, oleic acid (OA), stearic acid (SA) and cholesterol (CHOL).

Figures 2A-6A show the surface pressure-area per molecule isotherm for these lipids in water and in $0.1 \mathrm{~g} \cdot \mathrm{L}^{-1} \mathrm{LYS}$ aqueous subphase. Figures 2B-6B show the calculated elastic modulus, $\mathrm{C}_{\mathrm{s}}^{-1}$ (eq. 1), versus surface pressure obtained from these isotherms. The criteria that relate the elastic modulus values with the physical state of the monolayer is [45,46]: $\mathrm{C}_{\mathrm{s}}^{-1}<100 \mathrm{mN} \cdot \mathrm{m}^{-1}$ for liquid expanded (LE) state, $100-250 \mathrm{mN} \cdot \mathrm{m}^{-1}$ for liquid condensed (LC) state, $>250 \mathrm{mN} \cdot \mathrm{m}^{-1}$ for solid (S) state.

$$
C_{s}^{-1}=-A\left(\frac{d \pi}{d A}\right)_{T}
$$

Figure $2 \mathrm{~A}$ shows the surface pressure-area isotherms for DPPC in the absence and in the presence of LYS in the subphase. Figure 2B shows the elastic modulus obtained from these isotherms. A notable influence of LYS on DPPC monolayer behaviour is observed at low and moderate surface pressures. The lift off area increases in respect to DPPC, then a first inflection at around $8 \mathrm{mN} / \mathrm{m}$, usual for DPPC, occurs and a second inflection is observed at around $20 \mathrm{mN} / \mathrm{m}$. After

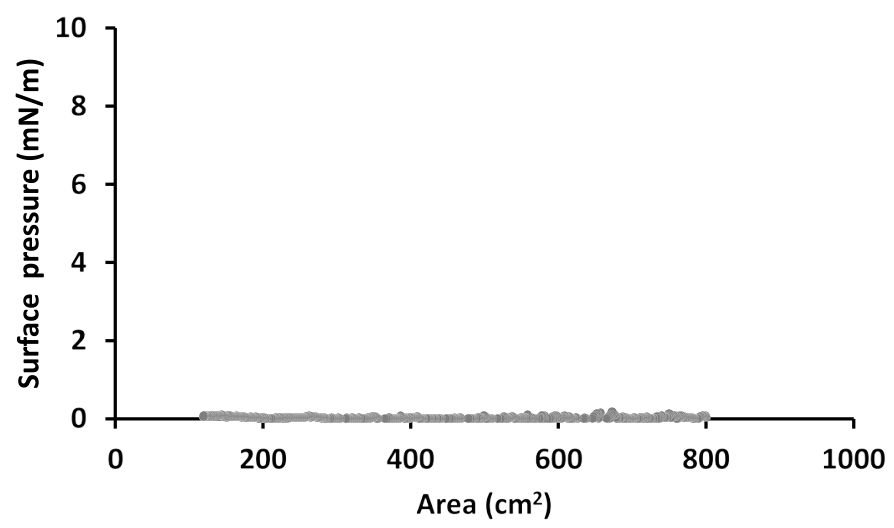

Figure 1. Surface pressure-area isotherm for $0.1 \mathrm{~g} \cdot \mathrm{L}^{-1} \mathrm{LYS}$ aqueous subphase.
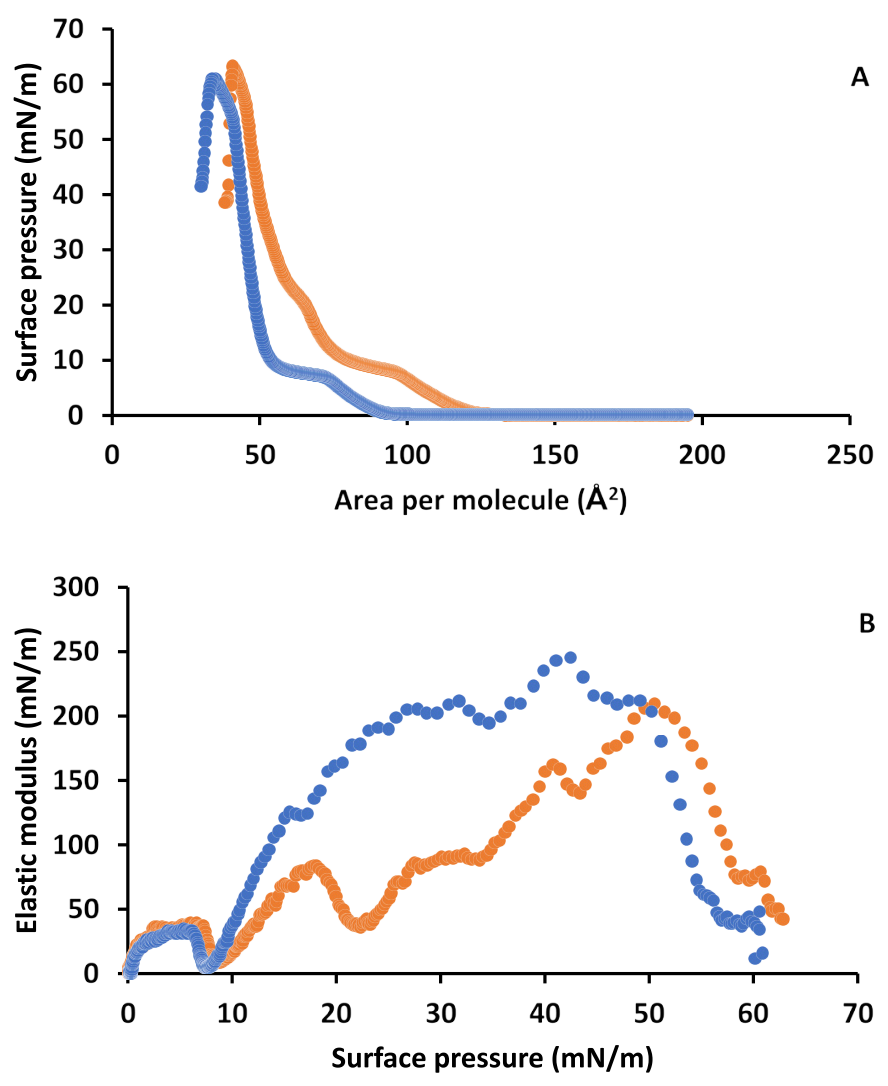

Figure 2. A) Surface pressure-area per molecule isotherm for DPPC on water (blue) and on $0.1 \mathrm{~g} \cdot \mathrm{L}^{-1} \mathrm{LYS}$ aqueous subphase (orange). B) Elastic modulus-surface pressure plots of the corresponding isotherms in A).

the second inflection the isotherm in presence of LYS gets closer to that in its absence, and the collapse surface pressure is practically the same, with only a small increase in the collapse area. In addition, the elastic modulus shows a decrease in presence of LYS in respect to what happens in its absence after the first inflection, with a phase change from a LE state to another LE state at the first inflection, according to the $\mathrm{C}_{\mathrm{S}}^{-1}$ values, that we are going to indicate as LE1 to LE2, and a phase change from LE2 to LC at the second inflection. DPPC in the absence of LYS shows the well stablished phase change from LE to LC at the inflection point around $8 \mathrm{mN} / \mathrm{m}$.

Fainerman et al. [47] studied another protein system and proposed that $\beta$-lactoglobulin penetration into a DPPC monolayer induces a first- 
order phase transition in the fluid-like monolayer, and after the phase transition point, condensed domains similar to those of pure DPPC are formed. These authors also indicate that the adsorbed protein induces the condensation of the pure DPPC compound: At the beginning, aggregation to condensed phase structures does not occur, induced by the penetrated species, and that the condensed phase consists only of the insoluble monolayer component.

Figure 3A shows the surface pressure-area isotherms for POPC in the absence and in the presence of LYS in the subphase. Figure 3B shows the elastic modulus obtained from these isotherms. It is observed a notable influence of LYS on POPC monolayer behaviour at low and moderate surface pressures. The lift off area increases and an inflection is observed at around $20 \mathrm{mN} / \mathrm{m}$. After the inflection, the isotherm in presence of LYS gets closer to that in its absence and the collapse point is practically the same. In addition, the elastic modulus shows a decrease in presence of LYS in respect to what happens in its absence, especially after the inflection, with a phase change from LE1 to LE2. POPC in the absence of LYS does not show a phase change.

Figure 4A shows the surface pressure-area isotherms for $\mathrm{OA}$ in the absence and in the presence of LYS in the subphase. Figure $4 \mathrm{~B}$ shows the elastic modulus obtained from these isotherms. It is observed a notable influence of LYS on OA monolayer behaviour at all surface pressures. The lift off area increases considerably and an inflection is observed at around $23 \mathrm{mN} / \mathrm{m}$. After the inflection, the isotherm in presence of LYS gets closer to that in its absence but with still an important area increase, and the collapse surface pressure and area are bigger in respect to those of OA in absence of LYS. In addition, the elastic modulus shows a notable decrease in presence of LYS, and at the inflection a phase change from LE1 to LE2 is observed. OA in absence of LYS does not shows a phase change, remaining in a LE state at all surface pressures.

Figure 5A shows the surface pressure-area isotherms for SA in the absence and in the presence of LYS in the subphase. Figure 5B shows the elastic modulus obtained from these isotherms. It is observed a notable influence of LYS on SA monolayer behaviour at low and moderate surface pressures. The lift off area increases considerably and an inflection is observed at around $23 \mathrm{mN} / \mathrm{m}$. After the inflection, the isotherm in presence of LYS gets closer to that in its absence, and practically superpose. The value of the surface pressure at the inflection is practically the same as that usual of SA, and the collapse point is practically the same. In addition, the elastic modulus shows a decrease in presence of LYS. Meanwhile SA shows a phase change from LC to $S$ at the inflection in the absence of LYS, a phase change from LE to LC and from LC to $\mathrm{S}$ is observed in the presence of LYS. This second phase change, at around $46 \mathrm{mN} / \mathrm{m}$, is visible in the elastic modulus plot more clearly than in the isotherm.

Figure $6 \mathrm{~A}$ shows the surface pressure-area isotherms for $\mathrm{CHOL}$ in the absence and in the presence of LYS in the subphase. Figure $6 \mathrm{~B}$ shows the elastic modulus obtained from these isotherms. It is observed a notable influence of LYS on CHOL monolayer behaviour at low and moderate surface pressures. The lift off area increases and an inflection is observed at around $23 \mathrm{mN} / \mathrm{m}$. After the inflection, the isotherm in presence of LYS gets closer to that in its absence and practically coincides, and the collapse point is practically the same. In addition, the elastic modulus shows a notable decrease in presence of LYS, in respect to what happens in its absence, with a phase change from LC to $\mathrm{S}$ at the inflection. CHOL in absence of LYS shows the well stablished $\mathrm{S}$ state at all surface pressures.
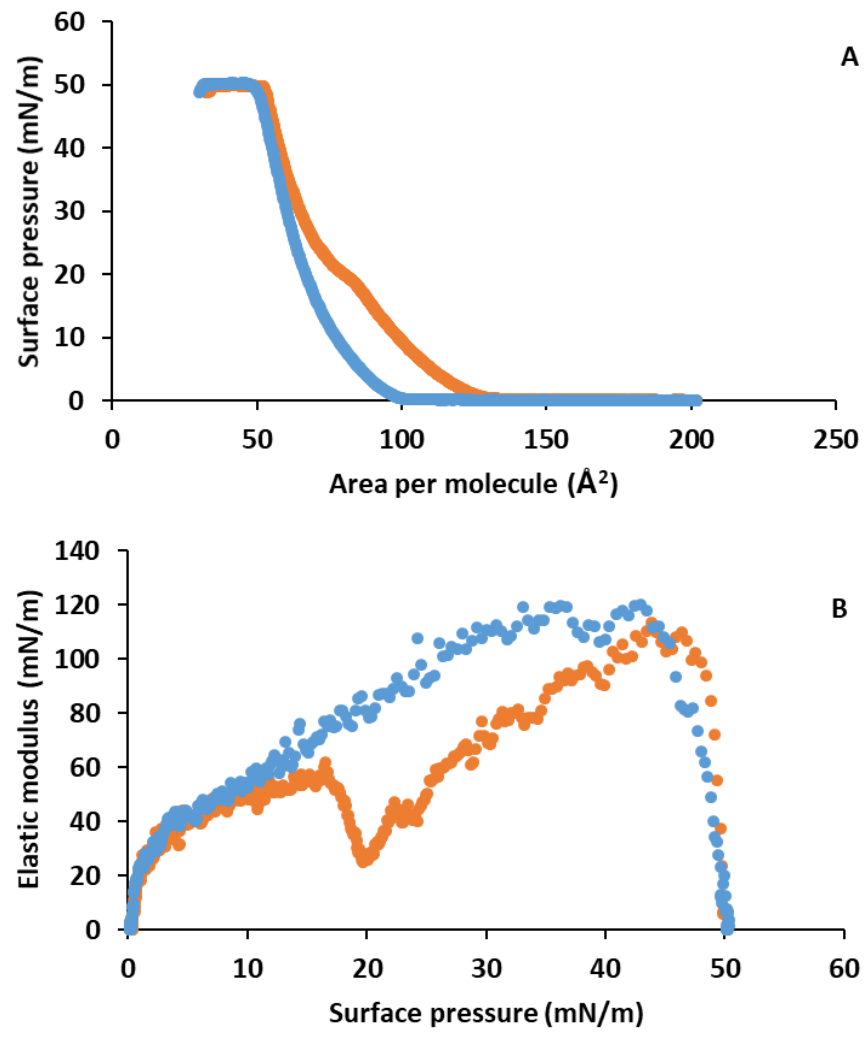

Figure 3. A) Surface pressure-area per molecule isotherm for POPC on water (blue) and on $0.1 \mathrm{~g} \cdot \mathrm{L}^{-1} \mathrm{LYS}$ aqueous subphase (orange). B) Elastic modulus-surface pressure plots of the corresponding isotherms in A).
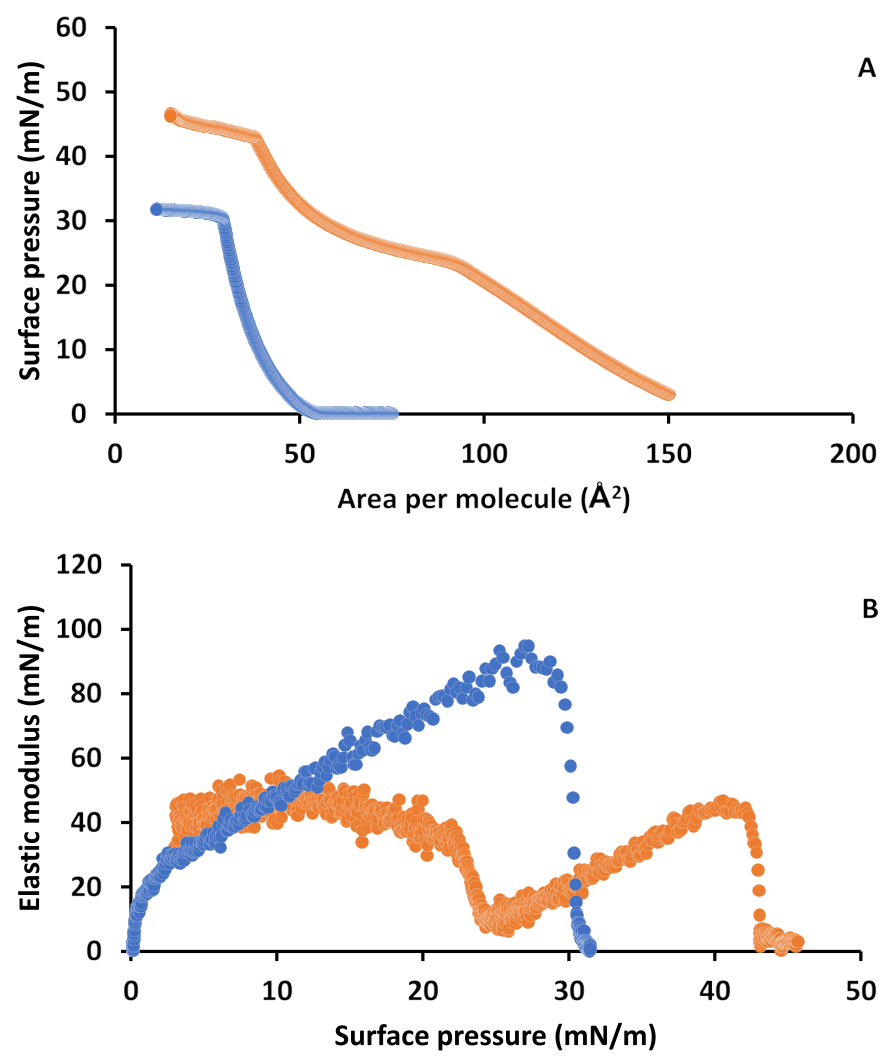

Figure 4. A) Surface pressure-area per molecule isotherm for $\mathrm{OA}$ on water (blue) and on $0.1 \mathrm{~g} \cdot \mathrm{L}^{-1} \mathrm{LYS}$ aqueous subphase (orange). B) Elastic modulus-surface pressure plots of the corresponding isotherms in A). 

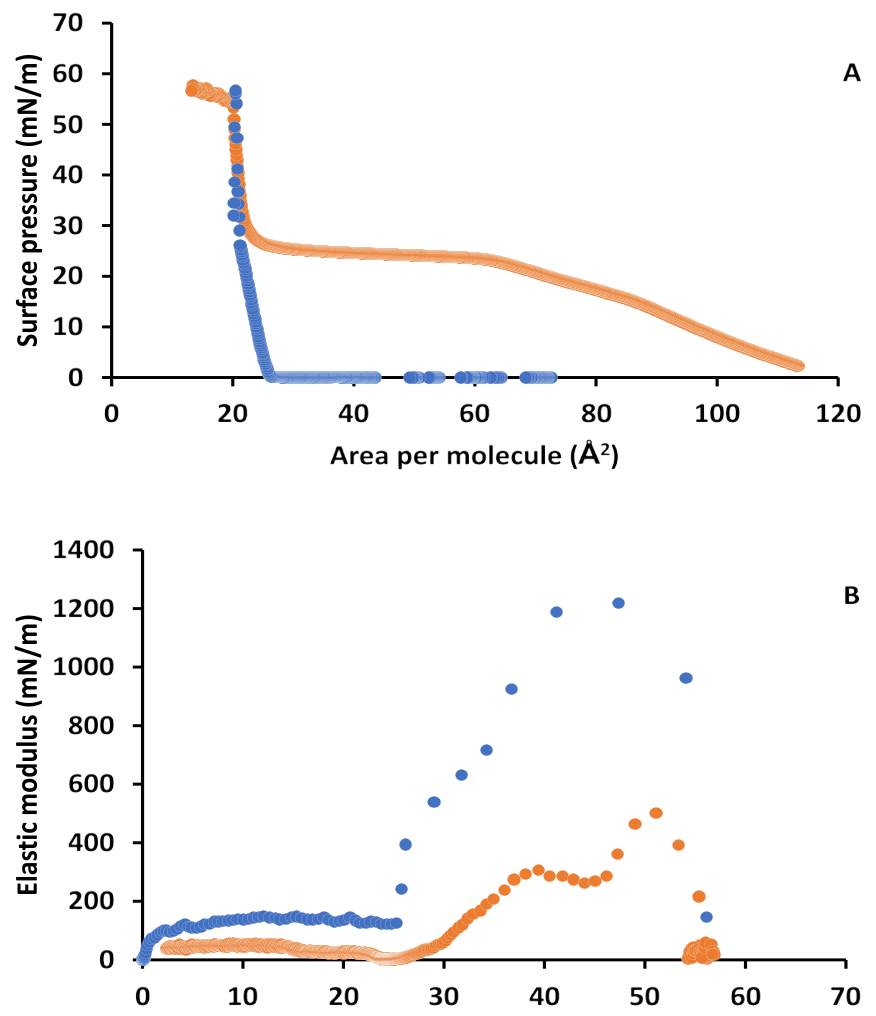

Figure 5. A) Surface pressure-area per molecule isotherm for SA on water (blue) and on $0.1 \mathrm{~g} \cdot \mathrm{L}^{-1} \mathrm{LYS}$ aqueous subphase (orange). B) Elastic modulus-surface pressure plots of the corresponding isotherms in A).
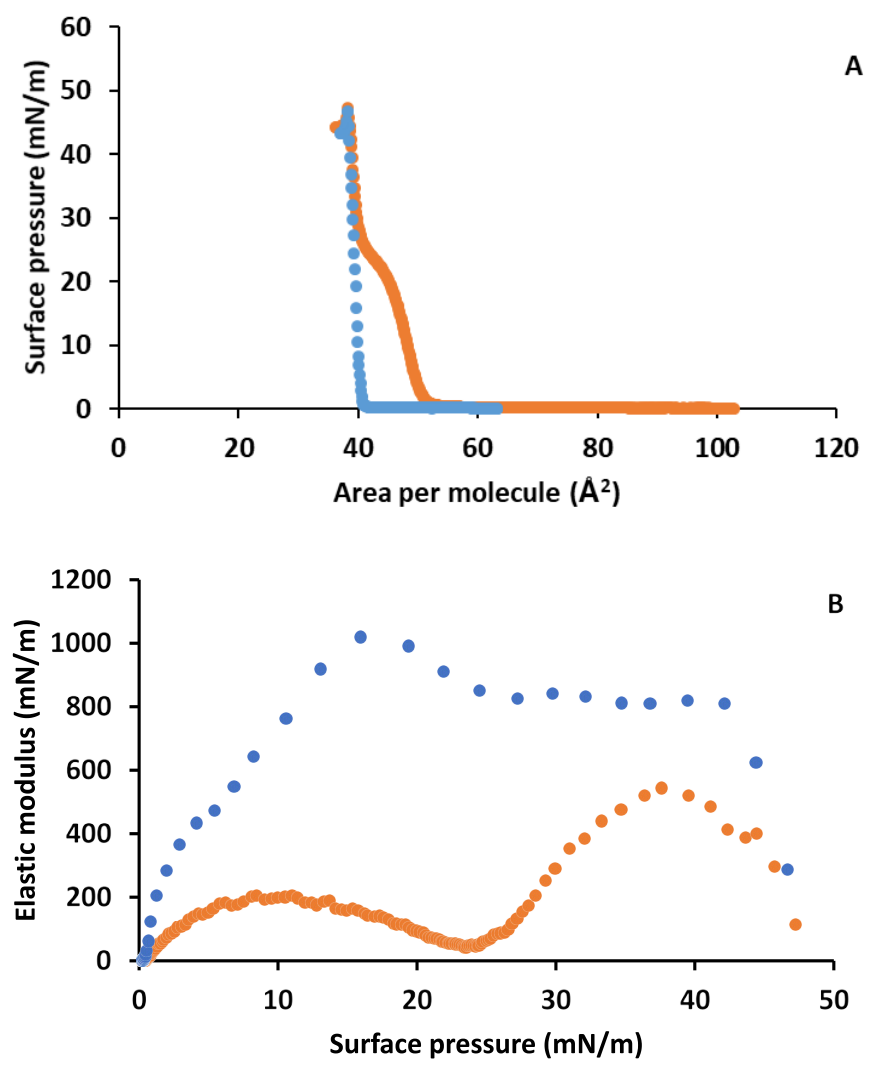

Figure 6. A) Surface pressure-area per molecule isotherm for $\mathrm{CHOL}$ in water (blue) and in $0.1 \mathrm{~g} \cdot \mathrm{L}^{-1} \mathrm{LYS}$ aqueous subphase (orange). B) Elastic modulus-surface pressure plots of the corresponding isotherms in A).

\section{Insertion experiments}

In order to see the affinity towards lipids, the ability of LYS to insert into monolayers of the lipids studied here was monitored by measuring variations in surface pressure, $\Delta \Pi$. For that different initial surface pressures, $\Pi_{i}$ were used and protein was injected in order to obtain a $0.1 \mathrm{~g} \cdot \mathrm{L}^{-1}$ concentration in the subphase. The general observed trend is that, greater the $\Pi_{\text {l }}$ lower the $\Delta \Pi$. That is, at a closer packing of the lipid at higher initial surface pressures corresponds a lower degree of incorporation of the protein into the monolayer (Figure 7).

The maximum insertion pressure (MIP), that is, the surface pressure above which the protein does not penetrate into the monolayer, was obtained by extrapolating the plot $\Delta \Pi$ vs $\Pi_{\mathrm{i}}$ to $\Delta \Pi=0 \mathrm{mN} \mathrm{m}^{-1}$ [37]. The MIP of proteins in lipid monolayers has been shown to be useful in characterizing protein adsorption and lipid specificity. Table 1 shows these values. It is noteworthy that the MIP values obtained for POPC and $\mathrm{OA}$ are larger than the membrane lateral pressure, which has been estimated to be in the range of $30-35 \mathrm{mN} / \mathrm{m}$.

The sequence on MIP, in the ascending order of values, is SA, DPPC, CHOL, POPC and OA. That means that OA allows the insertion till higher surface pressures, meanwhile SA allows insertion till the lowest surface pressure values. The values of MIP for DPPC, SA and $\mathrm{CHOL}$ are closer to the values of the inflection point observed in the isotherms in presence of LYS. This observation coincides with the fact that the isotherm becomes close to that in absence of LYS. It means that at this surface pressure, around $20-24 \mathrm{mN} / \mathrm{m}$, the expulsion of LYS should occur from the monolayer while compressing. A value of 20 $\mathrm{mN} / \mathrm{m}$ reported by Mudgil et al. [34] for DPPC is close to the value of MIP found by us.

For POPC the value of MIPП32.6 is higher than that of the inflection point but is close to the surface pressure where isotherms become closer. For OA the high value of MIP OA the high value of MIP44.6 is in 44.6 is in accordance with that observed in the isotherm, where the influence of LYS occurs even at high surfaces pressures. Miano et al. [1] reported that several proteins (lysozyme, lactoferrin, albumin,

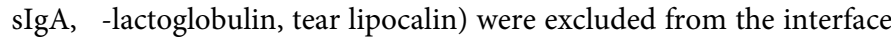
formed by Meibomian lipids, obtained from tear films, at a surface pressure above $30 \mathrm{mN} / \mathrm{m}$. Meibomian lipids are a complex mixture and only the MIP of POPC agrees with the later reported value, indicating that POPC could be a good model for Meibomian lipids.

Considering the physical states of the lipids in the monolayer, it is seen that LYS shows higher MIP for LE states (POPC, OA). These results indicate that LYS insertion is easier in monolayer films of those lipids that have unsaturated acyl chains, meanwhile the insertion is more difficult in those lipids that form more compact monolayers, for which the MIP is around $20-24 \mathrm{mN} / \mathrm{m}$. This value is similar to other published values with different proteins and phospholipids [33,37]. A value of $20.8 \mathrm{mN} / \mathrm{m}$ was observed for the MIP of rhodopsin in DDHAPC monolayers [33], and a similar value was observed for retinitis pigmentosa 2 with DDHA-PC monolayers [37]. Nevertheless, other values were observed for other protein-phospholipid systems [37,48].

Calvez et al. [37] proposed the eq. 2 to analyse the binding, insertion and interaction of proteins in lipid monolayers.

$$
\Pi_{e}=a \Pi_{i}+b
$$

Analysing the eq. 2 , that it is equivalent to $\Delta \Pi=(a-1) \Pi_{i}+b$, where $\Pi$ is the initial surface pressure and $\Pi_{e}$ the surface pressure after insertion, the values of $a$ and $b$ are obtained (Table 1). Value of $a>0$, being called the synergy factor, corresponds to favourable conditions for protein monolayer binding [37]. Value of $b$ is the extrapolation of $\Delta \Pi$ at zero 


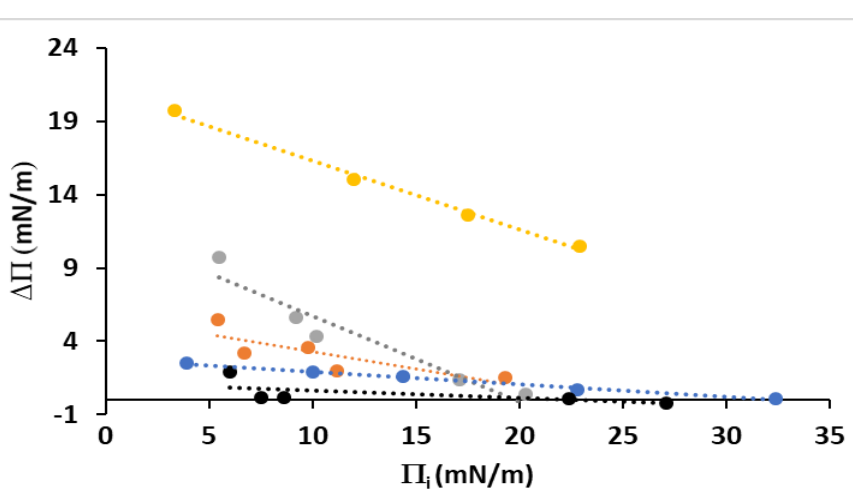

Figure 7. Surface pressure increase $(\Delta \Pi)$ in front of the lipid initial surface pressure $\left(\Pi_{i}\right)$, caused by LYS insertion on monolayers of several lipids: DPPC (black), POPC (blue), SA (grey), CHO (red), OA (yellow).

Table 1. Maximum insertion pressure (MIP) of LYS in lipid monolayers, and values of the parameters of eq. 2

\begin{tabular}{|l|c|c|c|c|c|}
\hline Lipid & DPPC & POPC & OA & SA & CHOL \\
\hline MIP & 22.6 & 32.6 & 44.6 & 19.8 & 24.0 \\
\hline$a$ & 0.947 & 0.914 & 0.529 & 0.417 & 0.766 \\
\hline$b$ & 1.20 & 2.79 & 21.01 & 11.55 & 5.61 \\
\hline $\begin{array}{l}\text { State in absence } \\
\text { of LYS }\end{array}$ & LE-LC & LE & LE & LC-S & S \\
\hline $\begin{array}{l}\text { State in presence } \\
\text { of LYS }\end{array}$ & LE1-LE2-LC & LE1-LE2 & LE1-LE2 & LE-LC-S & LC-S \\
\hline
\end{tabular}

surface pressure. The higher value of the parameter $a$ corresponds to DPPC, followed by POPC, CHOL, OA and SA. According to the value of $a$, the binding of LYS is more favourable for DPPC. On contrary, values of the parameter $b$ increase in the sequence DPPC, POPC, CHOL, SA and OA. Phospholipids present values of $a$ higher than those of fatty acids or CHOL, and the contrary occurs with the values of $b$. Thus, even DPPC and POPC allows the insertion, the effect on the increase of $\Pi$ is low. The highest effect is shown by OA.

The parameter $b$ must be described as the trend of the monolayer of modifying the protein surface activity [37]. When $b$ is larger than the protein surface tension, protein would insert within the lipid acyl chains. (This happens for SA, OA and CHOL, but no for DPPC and POPC). For low values of $b$, it can be postulated that protein would not be inserted more deeply than the polar headgroup of the lipid monolayer. In contrast when $b$ is high, protein would insert within the lipid acyl chain.

\section{Global Discussion}

Different behaviours have been observed about the influence of LYS on lipid monolayers depending on the lipid, but the isotherm shape in presence of LYS shows more differences with respect to that in the absence of LYS at low compressions for all lipids. This could indicate that at the first stages of compression, this one is more dependent on the protein. The pKa values for SA and OA have been reported to be 10.15 and 9.85, respectively [49], or also 9.0 for SA [49], even lower values were reported olderly (5.63 and 6.22 for SA and OA [50]). These values indicate that at the working $\mathrm{pH}$ of 5.0 these acids are present basically in the neutral form, but we have observed a noticeable influence of LYS on the fatty acids, OA and SA, at low compressions. As has been reported that the attractive electrostatic interaction seems to favour the insertion of LYS in the fatty acid monolayer [41] it is plausible to propose that in presence of lysozyme (isoelectric point $\mathrm{IP}=10.7[51])$, fatty acids deprotonated more easily acquiring negative charge and then interacting electrostatically with the positive charges of lysozyme. CHOL has no charge and zwitterion PCs have no neat charge. Miller et al. [40] says that the presence of only small amounts of ionic surfactants can significantly modify the structure of adsorbed proteins. With increasing amounts of ionic surfactants, an opposite effect is reached as due to hydrophobic interactions and the protein can be displaced from the interface due to competitive adsorption. In the presence of non-ionic surfactants, the adsorption layer is mainly formed by competitive adsorption and the only interaction is of hydrophobic nature. From a certain surfactant concentration, the interface is covered almost exclusively by the non-ionic surfactant.

When compressing SA, which forms compact monolayers, LYS is practically expelled at high surface pressures (the difference in area between isotherms in absence and in presence of LYS is very small, as well as the difference in the collapse). This expulsion is gradual with the phase change of SA. On the other hand, OA does not form monolayers as compact as SA and thus the expulsion of LYS is not so important (the difference in area between isotherms in absence and in presence of LYS is significant even at high surface pressures, and the collapse surface pressure increases in presence of LYS). And in the case of CHOL, it even forms compact monolayers, the insertion of LYS is notable, the difference in area between isotherms in absence and in presence of LYS is significant at low surface pressures but insignificant at high surface pressures, and the collapse surface pressure doesn't change. Thus, CHOL shows a behaviour more similar to SA than to OA. This could be due to the degree of compactness shown by SA and CHOL.

In the case of DPPC the expulsion of LYS is practically total at medium surface pressures (the difference in area between isotherms in absence and in presence of LYS is practically null, and the collapse surface pressure is the same). In the case of POPC there is also an expulsion practically total at medium surface pressures (the difference in area between isotherms in absence and in presence of LYS is also practically null, and the collapse surface pressure is also practically the same). Thus, even DPPC and POPC differs in one unsaturation and that POPC forms monolayers less compact than DPPC (due to the unsaturation present in one of the hydrocarbon chains), no much differences were observed for LYS influence in the isotherms. But the insertion experiments indicate more difficulty for LYS to insert in DPPC than in POPC, which is in agreement with the tendency of DPPC to form more compact monolayers than POPC, due to the presence of an unsaturation in the later. A lower lysozyme insertion for DPPC in respect to other phospholipids was also observed by Mudgil et al. [34], where PE, PS and PG phospholipids with unsaturations in the acyl chains were used. Moreover, PS and PG are anionic phospholipids.

DPPC and POPC are zwitterion phospholipids, and at a surface pressure around $33 \mathrm{mN} / \mathrm{m}$, close to the physiologic value of natural membranes, the effect of lysozyme on the phospholipid monolayer is practically null. Gorbenko et al. [41] studied the binding of lysozyme to phospholipid bilayers and found an influence of the content of anionic phospholipids on the binding of lysozyme. In our study, no anionic phospholipids were used, but ionisable anionic fatty acids, and these fatty acids are those that show a higher influence.

Comparing all the lipids at high surface pressures, SA and DPPC show similar behaviour due to the absence of unsaturations in the hydrocarbon chains, while OA and POPC show differences even the presence of an unsaturation. This fact could be explained as POPC also have a saturated chain that allows to form more compact monolayers in respect to OA. CHOL presents a specific behaviour which is more similar to that of SA and phospholipids than to that of OA. 
It is also seen that different sequences are obtained depending on the parameter if $a, b$ or MIP are used. The sequence, in the ascending order of values, is SA, DPPC, CHOL, POPC and OA for MIP; SA, OA, CHOL, POPC and DPPC for the parameter $a$; DPPC, POPC, CHOL, $\mathrm{SA}$ and OA for the parameter $b$. MIP is more related to compactness and physical state of the monolayer, and condensed monolayers provide lower MIP. The parameter $a$ is higher for phospholipids, meanwhile the parameter $b$ is lower for them, and thus the interaction of lysozyme is more favourable with the headgroups of PCs. As DPPC forms condensed monolayers, thus the insertion between acyl chains is not favoured, but for POPC the presence of the double bond makes the monolayer more fluid and the insertion of lysozyme is more favourable, even according to the parameter $b$ this insertion is near the headgroups. For SA and CHOL, that form condensed monolayers, the insertion is only possible at low surface pressures, and according to the parameter $b$ this insertion takes place within the acyl chains. OA presents a double bond in the acyl chain and forms the most fluid monolayer, and consequently the MIP is the highest and the parameter $b$ is also the highest, indicating the facility of lysozyme to insert within the acyl chains of OA.

\section{Conclusions}

According to surface pressure-area isotherms and elastic modulus plots, it is observed that lysozyme affects the lipid monolayer formation. This influence is more important till moderate surface pressures, but at high surface pressures there is expulsion of lysozyme out of the monolayer. This expulsion is more important for stearic acid, DPPC, POPC and cholesterol, meanwhile for oleic acid the isotherms indicate that a residual content of lysozyme remains in the monolayer. The sequence on Maximum Insertion Pressure (MIP) in the ascending order of values, is SA, DPPC, CHOL, POPC and OA. Even DPPC and POPC allows the insertion, the effect on the increase of $\Pi$ is low, and it can be postulated that in this case protein would not be inserted more deeply than the polar headgroup of the phospholipid monolayer. For $\mathrm{CHOL}$ and especially for SA and OA this insertion takes place between the acyl chains.

\section{Acknowledgements}

R. Raju thanks to the European Union and UPC for an Erasmus-K7 grant.

\section{References}

1. Miano F, Calcara M, Millar TJ, Enea V (2005) Insertion of tear proteins into a meibomian lipids film. Colloids Surf B Biointerfaces 44: 49-55. [Crossref]

2. Ulaganathan V, Retzlaff I, Won JY, Gochev G, Gehin-Delval C, et al. (2017) $\beta$-Lactoglobulin adsorption layers at the water/air surface: 1 . Adsorption kinetics and surface pressure isotherm: Effect of $\mathrm{pH}$ and ionic strength. Colloids Surf A 519: 153-160.

3. Boucher J, Trudel E, Méthot M, Desmeules P, Salesse C (2007) Organization, structure and activity of proteins in monolayers. Colloids Surf B Biointerfaces 58: 137-144. [Crossref]

4. Crawford NF, Micic M, Orbulescu J, Weissbart D, Leblanc RM (2015) Surface chemistry and spectroscopy of the $\beta$-galactosidase Langmuir monolayer. $J$ Colloid Interf Sci 453: 202-208. [Crossref]

5. Gálvez-Ruiz MJ (2017) Different approaches to study protein films at air/water interface. Adv Colloid Interface Sci 247: 533-542. [Crossref]

6. Gochev G, Retzlaff I, Aksenenko EV, Fainerman VB, Miller R (2013) Adsorption isotherm and equation of state for $\beta$-Lactoglobulin layers at the air/water Surface. Colloids Surf A 422: 33-38.

7. Ganzevles RA, Fokkink R, van Vliet T, Cohen Stuart MA, de Jongh HH (2008) Structure of mixed B-lactoglobulin/pectin adsorbed layers at air/water interfaces; a spectroscopy study. J Colloid Interf Sci 317: 137-147. [Crossref]
8. Liu W, Li S, Wang Z, Yan ECY, Leblanc RM (2017) Characterization of surface-active biofilm protein BsIA in self-assembling Langmuir monolayer at the air-water interface. Langmuir 33: 7548-7555. [Crossref]

9. Conde MM, Conde O, Trillo JM, Miñones J Jr (2011) How to obtain a well-spread monolayer of lysozyme at the air/water interfaces. J Colloid Interface Sci 361: 351360. [Crossref]

10. Sah BK, Kundu S (2017) Modification of hysteresis behaviours of protein monolayer and the corresponding structures with the variation of the protein surface charges. Colloids Surf B 159: 696-704. [Crossref]

11. Tihonov MM, Milyaeva OY, Noskov BA (2015) Dynamic surface properties of lysozyme solutions. Impact of urea and guanidine hydrochloride. Colloids Surf B Biointerfaces 129: 114-120. [Crossref]

12. Ayoub FP, Caseli L (2017) Controling the molecular architecture of lactase immobilized in Langmuir-Blodgett films of phospholipids to modulate the enzyme activity. Colloids Surf B Biointerfaces 150: 8-14. [Crossref]

13. Bourlieu C, Paboeuf G, Chever S, Pezennec S, Cavalier JF, et al. (2016) Adsorption of gastric lipase onto multicomponent model lipidmonolayers with phase separation. Colloids Surf B Biointerfaces 143: 97-106. [Crossref]

14. Caseli L, Crespilho FN, Nobre TM, Zaniquelli MED, Zucolotto V, et al. (2008) Using phospholipid Langmuir and Langmuir-Blodgett films as matrix for urease immobilization. J Colloid Interf Sci 319: 100-108.

15. Chernyssheva MG, Badun GA, Shnitko AV, Petrova VI, Ksenofontov AL (2018) Lysozyme-surfactant adsorption at the aqueous-air and aqueous-organic liquid interfaces as studied by tritium probe. Colloids Surf $A$ 537: 351-360.

16. Crawford NF, Leblanc RW (2014) Serum Albumin in 2D Langmuir monolayer study. Adv Colloid Interf Sci 207: 131-138. [Crossref]

17. Derde M, Nau F, Lechevalier V, Guérin-Dubiard C, Paboeuf G, et al. (2015) Native lysozyme and dry-heated lysozyme interactions with membrane lipid monolayers: Lateral reorganization of LPS monolayer, model of the Escherichia coli outer membrane. Biochem Biohyis Acta 1848: 174-183. [Crossref]

18. Derde M, Nau F, Guérin-Dubiard C, Lechevalier V, Paboeuf G, et al. (2015) Native and dry-heated lysozyme lysozyme interactions with membrane lipid monolayers: Lipid packing modifications of a phospholipid mixture, model of the Escherichia coli cytoplasmic membrane. Biochem Biohyis Acta 1848: 1065-1073. [Crossref]

19. Fragneto G, Alexandre S, Valleton JM, Rondelez F (2013) Competition for space between a protein and lipid monolayers. Colloids Surf B Biointerfaces 103: 416-421. [Crossref]

20. Le Guillou J, Ropers MH, Gaillard C, David-Briand E, van Leeuwen-Ibarrola J, et al. (2016) Sequestration of bovine seminal plasma proteins by different assemblies of phosphatidylcholine: A new technical approach. Colloids Surf B Biointerfaces 140 523-530. [Crossref]

21. Kamilya T, Pal P, Talapatra GB (2007) Interaction and incorporation of ovalbumin with stearic acid monolayer: Langmuir-Blodgett film formation and deposition. Colloids Surf B Biointerfaces 58: 137-144. [Crossref]

22. Kundu S, Matsuoka H, Seto H (2012) Zwitterionic lipid (DPPC)-protein (BSA) complexes at the air-water interface. Colloids Surf B Biointerfaces 93: 215-218. [Crossref]

23. Mady MM (2007) Biophysical studies on collagen-lipid interaction. J Biosci Bioeng 104: 144-148. [Crossref]

24. Mahato M, Pal P, Tah B, Talapatra GB (2012) Hemoglobin-phospholipid interaction and biocomposite formation at air/water interface. Colloids Surf A 414: 375-383.

25. Matyszewska D, Brzezińska K, Juhaniewicz J, Bilewicz R (2015) pH dependence of daunorubicin interactions with model DMPC:Cholesterol membranes. Colloids Surf B Biointerfaces 134: 295-303. [Crossref]

26. Nishimura SY, Magana GM, Ketelson HA, Fuller GG (2008) Effect of Lysozyme Adsorption on the Interfacial Rheology of DPPC and Cholesteryl Myristate Films. Langmuir 24: 11728-11733. [Crossref]

27. Ohno M, Toyota T, Nomoto T, Fujinami M (2015) Interfacial tension in adsorption of lysozyme onto a lipid monolayer formed at a water/chloroform interface. Colloids Surf $A$ 480: 85-90.

28. Sakamoto S, Nakahara H, Uto T, Shoyama Y, Shibata O (2013) Investigation of interfacial behavior of glycyrrhizin with a lipid raft model via a Langmuir monolayer study. Biochim Biophys Acta 1828: 1271-1283. [Crossref] 
29. Scholl FA, Caseli L (2015) Langmuir and Langmuir-Blodgett films of lipids and penicillinase: Studies on adsorption and enzymatic activity. Colloids Surf B Biointerfaces 126: 232-236. [Crossref]

30. de Araújo FT, Caseli L (2016) Rhodanese incorporated in Langmuir and LangmuirBlodgett films of dimyristoylphosphatidic acid: Physical chemical properties and improvement of the enzyme activity. Colloids Surf B Biointerfaces 14: 59-64. [Crossref]

31. Toimil P, Prieto G, Miñones J Jr, Trillo M, Sarmiento F (2012) Monolayer and Brewster angle microscopy study of human serum albumin-Dipalmitoylphosphatidylcholine mixtures at the air-water interface. Colloids Surf B Biointerfaces 92: 64-73. [Crossref]

32. Zanon NCM, Oliveira ON Jr, Caselli L (2012) Immobilization of uricase enzyme in Langmuir and Langmuir-Blodgett films of fatty acids: Possible use as a uric acid sensor. J Colloid Interf Sci 373: 69-74. [Crossref]

33. Sánchez-Martín MJ, Ramon E, Torrent-Burgués J, Garriga P (2013) Improved conformational stability of the visual $\mathrm{G}$ protein-coupled receptor rhodopsin by specific interaction with docosahexaenoic acid phospholipid. ChemBioChem 14: 639-644. [Crossref]

34. Mudgil P, Torres M, Millar TJ (2016) Adsorption of lysozyme to phospholipid and Meibomian lipid monolayer films. Colloids Surf B Biointerfaces 48: 128-137. [Crossref]

35. Maiti K, Bhattacharya SC, Moulik SP, Panda AK (2013) Effect of bovine serum albumin on the functionality and structure of catanionic surfactant at air-buffer interface. Mater Sci Eng C 33: 836-843.

36. Boisselier É, Demers É, Cantin L, Salesse C (2017) How to gather useful and valuable information from protein binding measurements using Langmuir lipid monolayers. $A d v$ Colloid Interf Sci 243: 60-76. [Crossref]

37. Calvez P, Demers E, Boisselier E, Salesse C (2011) Analysis of the contribution of saturated and polyunsaturated phospholipid monolayers to the binding of proteins. Langmuir 27: 1373-1379. [Crossref]

38. Fainerman VB, Lucassen-Reynders EH, Miller R (2003) Description of the adsorption behaviour of proteins at water/fluid interfaces in the framework of a two-dimensional solution model. Adv Colloid Interf Sci 106: 237-259. [Crossref]

39. Vollhardt D, Fainerman VB (2000) Penetration of dissolved amphiphiles into twodimensional aggregating lipid monolayers. Adv Colloid Interf Sci 86: 103-151. [Crossref]
40. Miller R, Fainerman VB, Makievski AV, Krägel J, Grigoriev DO, et al. (2000) Dynamics of protein and mixed protein surfactant adsorption layers at the water/fluid interface. Adv Colloid Interf Sci 86: 39-82. [Crossref]

41. Gorbenko GP, Ioffe VM, Kinnunen PKJ (2007) Binding of lysozyme to phospholipid bilayers: Evidence for protein aggregation upon membrane association. Biophys $J$ 93: 140-153. [Crossref]

42. Trusova V (2012) Modulation of physiological and pathological activities of lysozyme by biological membranes. Cell Mol Biol Letters 17: 349-375. [Crossref]

43. Kayal TA, Nappini S, Russo E, Berti D, Bucciantini M, et al. (2012) Lysozyme interaction with negatively charged lipid bilayers: protein aggregation and membrane fusion. Soft Matter 8: 4524-4534.

44. da Rocha Junior C, Caseli L (2017) Adsorption and enzyme activity of asparaginase at lipid Langmuir and Langmuir-Blodgett films. Mater Sci Eng C Mater Biol Appl 73: 579-584. [Crossref]

45. Torrent-Burgués J, Cea P, Giner I, Guaus E (2014) Characterization of Langmuir and Langmuir-Blodgett films of a octasubstituted zinc phthalocyanine. Thin Solid Films 556: 485-494.

46. Davies JT, Rideal EK (1993) Interfacial phenomena, Academic Press, Cambridge, MA USA.

47. Fainerman VB, Zhao J, Vollhardt D, Makievski AV, Li LB (1999) Dynamics of $\beta$-lactoglobulin penetration into Langmuir monolayers of $2 \mathrm{D}$ condensating phospholipid. J Phys Chem B 103: 8998-9007.

48. Calvez P, Bussières S, Eric Demers, Salesse C (2009) Parameters modulating the maximum insertion pressure of proteins and peptides in lipid monolayers. Biochimie 91: 718-733. [Crossref]

49. Kanicky JR, Shah DO (2002) Effect of degree, type, and position of unsaturation on the pKa of long-chain fatty acids. J Colloid Interface Sci 256: 201-207. [Crossref]

50. Tomoaia-Cotisel M, Zsako J, Mocanu A, Lupea M, Chifu E (1987) Insoluble mixed monolayers: III. The ionization characteristics of some fatty acids at the air/wáter interface. J Colloid Interf Sci 117: 464-476.

51. You SJ, Udeniwe CC, Aluko RE, Wu J (2010) Multifunctional peptides from egg white lysozyme. Food Res Internat 43: 848-855.

Copyright: (C2019 Torrent-Burgués J. This is an open-access article distributed under the terms of the Creative Commons Attribution License, which permits unrestricted use, distribution, and reproduction in any medium, provided the original author and source are credited. 